\title{
On Two-machine Flow Shop Scheduling
}

\author{
Lin Chen · Wen-Chang Luo • Guo-Chuan Zhang
}

Received: 4 May 2014/Revised: 5 August 2014/Accepted: 11 August 2014/

Published online: 31 August 2014

(C) Operations Research Society of China, Periodicals Agency of Shanghai University, and SpringerVerlag Berlin Heidelberg 2014

\begin{abstract}
In this note, we revisit the classical two-machine flow shop scheduling problem. A linear time approximation scheme is presented. For an online version with rejection, we propose best possible online algorithms.
\end{abstract}

Keywords Flow shop - Linear time - Online algorithm

\section{Introduction}

In the classical two-machine flow shop scheduling problem, we are given a set of jobs $J=\left\{J_{1}, J_{2}, \cdots, J_{n}\right\}$ and two machines $M_{1}$ and $M_{2}$. Each job $J_{i}$ must first be executed on $M_{1}$ and then executed on $M_{2}$. The processing times are $p_{i}$ 's and $q_{i}$ 's, respectively. The goal is to assign the jobs to the machines so that the makespan is minimized. Following the 3-field notation of Graham et al. [4], this problem is known as $F 2 \| C_{\max }$. Johnson [6] proposes an elegant algorithm, called Johnson's rule, which runs in $O(n \log n)$ time.

In a variant where jobs can be rejected, each job $J_{i}$ has a penalty $r_{i}$ in addition to processing times, and the goal is to minimize the makespan of the accepted jobs plus the total penalty of the rejected jobs. This problem is denoted as $F 2 \mid$ rej $\mid C_{\max }^{A}+$

\footnotetext{
L. Chen · G.-C. Zhang $(\bowtie)$

College of Computer Science, Zhejiang University, Hangzhou 310027, China

e-mail: zgc@zju.edu.cn

L. Chen

e-mail: chenlin198662@gmail.com

W.-C. Luo

Faculty of Science, Ningbo University, Ningbo 315211, China

e-mail: luowenchang@163.com
} 
$\sum_{J_{i} \in \mathbb{R}} r_{i}$, where $A$ and $\mathbb{R}$ are the sets of accepted jobs and rejected jobs, respectively. Shabtay and Gasper [7] show the NP-hardness and propose a 2-approximation algorithm as well as a fully polynomial time approximation scheme (FPTAS).

At the age of big data, polynomial time algorithms are too costly for many realworld problems if their running time is superlinear. To this end, linear time algorithms receive much attention to deal with optimization problems even when they are polynomially solvable. One of the well-studied problems is the matching problem $[3,5]$. Along this line, we first revisit Johnson's rule, based on which we present a linear time approximation scheme for $F 2 \| C_{\max }$. This scheme shows the tradeoff between the running time and the accuracy.

Then we consider the online version of problem $F 2 \mid$ rej $\mid C_{\max }^{A}+\sum_{J_{i} \in \mathbb{R}} r_{i}$. In the online setting, jobs arrive one by one, and their information is known upon the arrival. We deal with two scenarios. In the first scenario, upon arrival of a job, we need to immediately decide whether the job is accepted or not, and where/when to schedule, if yes. In the second scenario, we only need to decide the acceptance of an incoming job, while the scheduling of all accepted jobs can be done as long as no more jobs show up. For both models, the decisions must be made without any information about future jobs. Best possible online algorithms with competitive ratio of 2 and $(1+\sqrt{5}) / 2$ are designed, respectively.

\section{A Linear PTAS}

In this section, we consider two-machine flow shop scheduling with an aim at designing linear time algorithms. Clearly, the main running time in Johnson's rule is sorting which incurs nonlinear time. Thus, as long as we do not execute the sorting operation, but only execute the partition operation, we can obtain a linear time algorithm. Based on the above idea, a linear time algorithm can be described as follows.

\section{Algorithm $H_{1}$}

Step 1. Partitioning the jobs into two sets $S_{1}$ and $S_{2}$, where $S_{1}$ is the set of jobs satisfying $p_{i} \leqslant q_{i}$ while $S_{2}$ is the set of jobs satisfying $p_{i}>q_{i}$.

Step 2. Schedule all the jobs in $S_{1}$ before all the jobs in $S_{2}$ in an arbitrary order.

Step 3. Process jobs as early as possible in the order constructed in Step 2 on both machines.

Theorem 2.1 For problem $F 2 \| C_{\max }$, Algorithm $H_{1}$ is a linear time algorithm with a worst-case ratio of $3 / 2$.

Proof It is clear that Algorithm $H_{1}$ runs in linear time. Without loss of generality, assume that the job order according to Algorithm $H_{1}$ is $J_{1}, J_{2}, \cdots, J_{n}$. Given this schedule, define the critical job as the last one whose completion time on the first machine is equal to its starting time on the second machine. The makespan can thus be determined by 


$$
C_{\max }=p_{1}+p_{2}+\cdots+p_{i}+q_{i}+q_{i+1}+q_{n},
$$

where job $J_{i}$ is the critical job. Let $C_{\max }^{*}$ denote the optimal value. Recall that

$$
C_{\max }^{*} \geqslant \max \left\{\sum_{i} p_{i}, \sum_{i} q_{i}, \max _{i}\left\{p_{i}+q_{i}\right\}\right\} .
$$

Depending on job $J_{i}$ in $S_{1}$ or $S_{2}$, the following two cases are considered.

Case 1: $J_{i} \in S_{1}$. Clearly we have $p_{k} \leqslant q_{k}, k=1,2, \cdots, i$. Thus,

$$
\begin{aligned}
C_{\max } & \leqslant p_{1}+p_{2}+\cdots+p_{i}+q_{i}+q_{i+1}+\cdots+q_{n} \\
& \leqslant\left(q_{1}+q_{2}+\cdots+q_{i}+q_{i+1}+\cdots+q_{n}\right)+p_{i} \\
& \leqslant C_{\max }^{*}+\frac{1}{2} C_{\max }^{*} \\
& \leqslant \frac{3}{2} C_{\max }^{*}
\end{aligned}
$$

Case 2: $J_{i} \in S_{2}$. The proof is similar to Case 1 .

To show the bound is tight, consider the following instance $J=\left\{J_{1}, J_{2}\right\}$, where $p_{1}=\varepsilon, q_{1}=1, p_{2}=1, q_{2}=1+\varepsilon$, where $\varepsilon$ is an arbitrarily small positive number. The schedule by Algorithm $H_{1}$ may be $\pi=\left(J_{2}, J_{1}\right)$, while the optimal schedule is $\pi^{*}=\left(J_{1}, J_{2}\right)$. Then

$$
\frac{C_{\max }}{C_{\max }^{*}}=\frac{3+\varepsilon}{2+2 \varepsilon} \rightarrow \frac{3}{2}(\varepsilon \rightarrow 0) .
$$

Although Algorithm $H_{1}$ runs in linear time, the approximation ratio is not satisfactory. Note that the large jobs in $S_{1}$ and $S_{2}$ play an important role. An intuitive way is to give a special treatment for large jobs. Along this line, we propose a linear time approximation scheme.

\section{Algorithm $\mathrm{H}_{2}$}

Step 1. The same as Algorithm $H_{1}$, partition the jobs into two sets $S_{1}$ and $S_{2}$.

Step 2. Given $\varepsilon>0$, select the largest $1 / \varepsilon$ jobs from $S_{1}$ in terms of the processing time on machine 1 and the largest $1 / \varepsilon$ jobs from $S_{2}$ in terms of the processing time on machine 2 by using the linear time selection algorithm proposed by Blum et al [1]. The selected job sets are $\mathbb{R}_{1}$ and $\mathbb{R}_{2}$, respectively.

Step 3. First schedule the jobs of $S_{1} \backslash \mathbb{R}_{1}$ in an arbitrary order, then the jobs of $\mathbb{R}_{1}$ in the non-decreasing order of $p_{i}$ 's, followed by the jobs of $\mathbb{R}_{2}$ in the nonincreasing order of $q_{i}$ 's, finally schedule the jobs of $S_{2} \backslash \mathbb{R}_{2}$ in an arbitrary order.

Step 4. Process the ordered jobs as early as possible.

Theorem 2.2 For the problem F2 $\| C_{\max }$, Algorithm $H_{2}$ runs in $O\left(\frac{n}{\varepsilon}+\frac{1}{\varepsilon} \log \frac{1}{\varepsilon}\right)$ time with a worst-case ratio of $(1+\varepsilon)$ and thus it is a linear time approximation scheme for any given $\varepsilon>0$. 
Proof Again, without loss of generality, assume that the jobs are ordered as $J_{1}, J_{2}, \cdots, J_{n}$, and the makespan can be represented as

$$
C_{\max }=p_{1}+p_{2}+\cdots+p_{i}+q_{i}+q_{i+1}+q_{n},
$$

where job $J_{i}$ is the critical job. Let $C_{\max }^{*}$ denote the optimal value. We distinguish three cases for $J_{i}$.

Case 1: $J_{i} \in S_{1} \backslash \mathbb{R}_{1}$. Clearly we have $p_{k} \leqslant q_{k}, k=1,2, \cdots, i$. Since $C_{\max }^{*} \geqslant$ $\sum_{j \in \mathbb{R}_{1}} p_{j}$ and $p_{i} \leqslant\left(\sum_{j \in \mathbb{R}_{1}} p_{j}\right) /(1 / \varepsilon)$,

$$
\begin{aligned}
C_{\max } & \leqslant p_{1}+p_{2}+\cdots+p_{i}+q_{i}+q_{i+1}+\cdots+q_{n} \\
& \leqslant\left(q_{1}+q_{2}+\cdots+q_{i}+q_{i+1}+\cdots+q_{n}\right)+p_{i} \\
& \leqslant C_{\max }^{*}+\varepsilon C_{\max }^{*} \\
& \leqslant(1+\varepsilon) C_{\max }^{*}
\end{aligned}
$$

Case 2: $J_{i} \in S_{2} \backslash \mathbb{R}_{2}$. The proof is similar to Case 1 .

Case 3: $J_{i} \in \mathbb{R}_{1} \cup \mathbb{R}_{2}$. In this case, we compare with an optimal schedule $\sigma^{*}$ by the Johnson's rule. In $\sigma^{*}$, the global order of the four job groups is still $\left(S_{1} \backslash \mathbb{R}_{1}, \mathbb{R}_{1}, \mathbb{R}_{2}, S_{2} \backslash \mathbb{R}_{2}\right)$, while both $S_{1} \backslash \mathbb{R}_{1}$ and $S_{2} \backslash \mathbb{R}_{2}$ are well sorted. Consider each job $J_{j} \in \mathbb{R}_{1} \cup \mathbb{R}_{2}$ in $\sigma^{*}$ (including job $J_{i}$ ). Its completion time on machine 1 is $\sum_{k=1}^{j} p_{k}$, which is not greater than its start time on machine 2. Thus,

$$
C_{\max }^{*} \geqslant \sum_{k=1}^{j-1} p_{k}+p_{j}+q_{j}+\sum_{l=j+1}^{n} q_{l} .
$$

It shows that the schedule constructed by Algorithm $\mathrm{H}_{2}$ is optimal.

The running time of Algorithm $\mathrm{H}_{2}$ is mainly decided by Step 2 which takes $O\left(\frac{n}{\varepsilon}+\frac{1}{\varepsilon} \log \frac{1}{\varepsilon}\right)$ time. Thus Algorithm $H_{2}$ can be implemented in $O\left(\frac{n}{\varepsilon}+\frac{1}{\varepsilon} \log \frac{1}{\varepsilon}\right)$ time.

\section{Online Scheduling with Rejection}

In this section, we first deal with a semi-online version for problem $F 2 \mid$ rej $\mid C_{\text {max }}^{A}+\sum_{J_{i} \in \mathbb{R}} r_{i}$. Consider the following scenario. Jobs arrive one by one. Upon arrival of a job, we have to immediately decide whether to reject it or not. However, all accepted jobs can be scheduled until no jobs show up. In other words, the problem consists of two phases. In the first phase, acceptance decision is made in an online fashion, while in the second phase, scheduling all accepted jobs can be done in the off-line way.

Let $\phi=(1+\sqrt{5}) / 2$, and let $A l g$ be any online algorithm. We show that the competitive ratio of $A l g$ is at least $\phi$ by constructing an instance. Denote a job as a triple $\left(p_{i}, q_{i}, r_{i}\right)$. The first job is $(\phi, 0,1)$. If $A l g$ accepts this job, the game is immediately over. In this case, the total cost is $\phi$ while in the optimal solution this job is rejected with a cost of one. If $A l g$ rejects the first job, then the second job $(0, \phi, 1)$ arrives. Again if Alg accepts this job, then no more jobs come. The resulting cost is $1+\phi$, while the optimal solution accepts both jobs with a cost $\phi$. 
Notice that the objective value of $A l g$ is $1+\phi$, while the optimum value is $\phi$, the ratio is still $\phi$. If $A l g$ rejects both jobs then the third job $(\phi, \phi,+\infty)$ comes. Alg has to accept this job and its objective value becomes $2+2 \phi$. As the optimum solution accepts all jobs and the optimum value is $2 \phi$, again the ratio is $\phi$.

Next, we propose a semi-online algorithm with the competitive ration $(1+\sqrt{5}) / 2$. We distinguish jobs as follows. For any job $J_{i}$, if $\left(p_{i}+q_{i}\right) / r_{i}<\phi$, it is a valuable job, otherwise it is a lost job.

Algorithm $H_{3}$ : Accept all valuable jobs and reject all lost jobs. Schedule the accepted jobs with Johnson's rule.

For any instance, we try to compare the online algorithm $\mathrm{H}_{3}$ and an optimal (offline) algorithm. Based on their schedules, we classify all jobs into four groups. Let $G_{1}$ consist of the jobs accepted by both $H_{3}$ and the optimal schedule, $G_{2}$ be the group of jobs accepted by the optimal schedule while rejected by $\mathrm{H}_{3}, \mathrm{G}_{3}$ denote the group of jobs accepted by $\mathrm{H}_{3}$ but rejected by the optimal schedule, and finally $G_{4}$ contains those jobs rejected by both. We use $C_{\max }(G)$ to denote the makespan of jobs in $G$ scheduled by Johnson's rule. We have the following lemma.

Lemma 3.1 $C_{\max }\left(G_{1}\right)+\sum_{i \in G_{2}} r_{i} \leqslant \phi \cdot C_{\max }\left(G_{1} \cup G_{2}\right)$.

Proof Let $L_{1}=\sum_{i \in G_{1}}\left(p_{i}+q_{i}\right)$ and $L_{2}=\sum_{i \in G_{2}}\left(p_{i}+q_{i}\right)$. Note that the optimal schedule accepts all jobs of $G_{1} \cup G_{2}$. It is obvious that $C_{\max }\left(G_{1} \cup G_{2}\right) \geqslant$ $\left(L_{1}+L_{2}\right) / 2 \geqslant\left(C_{\max }\left(G_{1}\right)+L_{2}\right) / 2$. On the other hand, $L_{2} \geqslant \phi \sum_{i \in G_{2}} r_{i}$. If $L_{2} \leqslant C_{\max }\left(G_{1}\right)$, we have

$$
\begin{aligned}
C_{\max }\left(G_{1}\right)+\sum_{i \in G_{2}} r_{i} & \leqslant C_{\max }\left(G_{1}\right)+L_{2} / \phi \leqslant(1+1 / \phi) C_{\max }\left(G_{1}\right) \\
& \leqslant \phi \cdot C_{\max }\left(G_{1} \cup G_{2}\right) .
\end{aligned}
$$

If $L_{2}>C_{\max }\left(G_{1}\right)$, then

$$
\begin{aligned}
C_{\max }\left(G_{1}\right)+\sum_{i \in G_{2}} r_{i} & \leqslant C_{\max }\left(G_{1}\right)+L_{2} / \phi \\
& \leqslant \phi \cdot\left(C_{\max }\left(G_{1}\right)+L_{2}\right) / 2 \\
& \leqslant \phi \cdot C_{\max }\left(G_{1} \cup G_{2}\right) .
\end{aligned}
$$

The proof is completed.

Based on the above lemma, we have the following theorem.

Theorem 3.2 Algorithm $\mathrm{H}_{3}$ has a competitive ratio of $(1+\sqrt{5}) / 2$.

Proof Let $Z$ denote the cost of algorithm $H_{3}$ and $O P T$ denote the optimal cost. Then, we have

$$
Z \leqslant C_{\max }\left(G_{1}\right)+\sum_{i \in G_{3}}\left(p_{i}+q_{i}\right)+\sum_{i \in G_{2} \cup G_{4}} r_{i}
$$

while 


$$
O P T=C_{\max }\left(G_{1} \cup G_{2}\right)+\sum_{i \in G_{3} \cup G_{4}} r_{i} .
$$

Clearly, $\sum_{i \in G_{3}}\left(p_{i}+q_{i}\right) \leqslant \phi \sum_{i \in G_{3}} r_{i}$. Combining with Lemma 3.1, we have

$$
\begin{aligned}
Z & \leqslant C_{\max }\left(G_{1}\right)+\sum_{i \in G_{2}} r_{i}+\phi \sum_{i \in G_{3} \cup G_{4}} r_{i} \\
& \leqslant \phi C_{\max }\left(G_{1} \cup G_{2}\right)+\phi \sum_{i \in G_{3} \cup G_{4}} r_{i} \\
& =\phi O P T .
\end{aligned}
$$

Now we turn to the online version. Upon arrival of a job, we have to decide whether to reject or accept it. If accepted, it must be immediately scheduled. Note that if all rejection penalty is infinite, the problem becomes the traditional flow shop problem. Chen and Woeginger [2] showed a lower bound of two for online twomachine flow shop scheduling, that could be achieved by a naive online algorithm. The lower bound remains true for our problem. Namely, an online algorithm does not exist with a competitive ratio strictly better than two. In the following, we present a very simple online algorithm that can reach this bound. Similar as the semi-online case, a job $J_{i}$ is called valuable if $p_{i}+q_{i}<r_{i}$, otherwise it is called lost.

Algorithm $H_{4}$ : Upon arrival of a job, if it is a lost job, reject it immediately; otherwise, accept and schedule it as early as possible.

Theorem 3.3 Algorithm $\mathrm{H}_{4}$ is a 2-competitive online algorithm.

Proof We classify the jobs into four groups as before. Clearly, any optimal algorithm does not reject a valuable job. It shows that $G_{3}=\emptyset$. Let $Z\left(G_{1}\right)$ be the makespan by algorithm $H_{4}$. Let $Z$ be the cost of the schedule by algorithm $H_{4}$, and $O P T$ be the optimal cost, respectively. We have

$$
\begin{aligned}
Z & =Z\left(G_{1}\right)+\sum_{i \in G_{2}} r_{i}+\sum_{i \in G_{4}} r_{i} \\
& \leqslant 2 \sum_{i \in G_{1} \bigcup G_{2}}\left(p_{i}+q_{i}\right) / 2+\sum_{i \in G_{4}} r_{i} \\
& \leqslant 2 \mathrm{OPT} .
\end{aligned}
$$

The theorem is proved.

Acknowledgments The authors would like to thank the two anonymous referees for their valuable comments that help improve the presentation of this paper.

\section{References}

[1] Blum, M., Floyd, R.W., Pratt, V., Rivest, R.L., Tarjan, R.E.: Time bounds for selection. J. Comput. Syst. Sci. 7, 448-461 (1973) 
[2] Chen, B., Woeginger, G.J.: A study of on-Line scheduling two-stage shops. In: Du, D.-Z., Pardalo, P.M. (eds.) Minimax and Applications, pp. 97-107. Kluwer Academic Publishers, Dordrecht (1995)

[3] Drake Vinkemeier, D.E., Hougardy, S.: A linear-time approximation algorithm for weighted matchings in graphs. ACM Trans. Algorithms 1, 107-122 (2005)

[4] Graham, R.L., Lawler, E.L., Lenstra, J.K., Kan, A.H.G.R.: Optimization and approximation in deterministic sequencing and scheduling: a survey. Ann. Discrete Math. 5, 287-326 (1979)

[5] Hougardy, S.: Linear Time Approximation Algorithms for Degree Constrained Subgraph Problems. Research trends in combinatorial optimization, pp. 185-200. Springer, Berlin (2009)

[6] Johnson, S.M.: Optimal two-and-three-stage production schedules with setup times included. Nav. Res. Logist. 1, 61-68 (1954)

[7] Shabtay, D., Gasper, N.: Two-machine flow-shop scheduling with rejection. Comput. Oper. Res. 39, 1087-1096 (2012) 